\title{
Prediction of Antigenic Epitope Patches on Protein Surface Using Antigen Structure Information and Support Vector Machine
}

\author{
Khale d A Hass an ${ }^{1, *}$, Amr Badr², Mostafa Abdel-Azim ${ }^{3}$ \\ ${ }^{1}$ Department of Computer Science, Arab Academy for Science, Technology and Maritime Transport, Cairo, Egypt \\ ${ }^{2}$ Department of Computer Science, Faculty of Computers and Information, Cairo University, Cairo, Egypt \\ ${ }^{3}$ Department of Information Systems, Arab Academy for Science, Technology and Maritime Transport, Cairo, Egypt
}

\begin{abstract}
Identification of antigen-antibody interacting sites is an important task for vaccine design, and hence reliable computer based prediction methods are highly desirable. The prediction performances of the current existing methods to predict the conformational B-cell ep itope residues are still not satisfying and rema in far from ideal. This is a new approach in the area of vaccine development to predict the antigenic surface patches that hold the majority number of epitope residues in the surface of the antigen protein structure. The proposed method is a support vector machine based model to predict the epitope patches in the antigen structures by combining the accessible surface area and B-factor structural features. The Predictions are made for the known structures of benchmark dataset after removing antigens sequence redundancy where no two antigen sequences have more than $40 \%$ sequence identity. The predictions are success ful for $70 \%$ of the antigen structure chains of the benchmark dataset. We compared the prediction performance of our model with a protein protein interaction prediction server "Sharp2" using the same antigen structures of the benchmark dataset and observed that our model outperforms on Sharp 2 by more than $40 \%$ accuracy. Th is paper demonstrates that the identification of the antigenic determinant sites in the protein surface using the antigen structural information outperforms the traditional protein-protein interaction algorith $\mathrm{ms}$ to predict the interacting sites in the antigen protein surface. It provides a new approach for the scientists to only use the predicted antigenic epitope surface patch from the target antigen structure in vaccine development rather than using the predicted epitope residues. A web server "PatchTope" has been developed for predicting antigenic epitope surface patches on an antigen protein structure surface and is available at http://www.fci.cu.edu.eg:8080/PatchTope/.
\end{abstract}

Keywords Surface Patch, Conformational B-cell Epitopes, Support Vector Machine, B-Factor, Relative Solvent Accessibility

\section{Introduction}

Vaccine design is the process of creating drug (vaccine) to stimulate adaptive immunity to a disease. Vaccine can either be live attenuated (weakened) forms of pathogens (bacteria or viruses), killed or inactivated forms of these pathogens, or a refiner material such as proteins. Evolution of weakened pathogens can be one of the potential safety problems raised from such vaccines [1]. In order to overcome on such safety problems, the subunit vaccine is introduced. Subunit vaccine is produced from a specific portion of the protein antigen or virus separated from the pathogenic organism called epitope.

* Corresponding author:

khaled.hassan.edu@gmail.com(Khaled A Hassan)

Published online at http://journal.sapub.org/bioinformatics

Copyright (C) 2012 Scientific \& Academic Publishing. All Rights Reserved
B-cell epitopes are segments of the antigen molecules recognized by antibodies or B-cells. They are classified into two groups: continuous and discontinuous. A continuous (linear) epitopes are short segment of continuous amino acid sequence fragment of a protein [2] while a discontinuous (conformational) epitopes are composed of a bundle of amino acid residues of a protein antigen that are far away from each other in the primary sequence of the antigen but are brought to close proximity within the folded protein structure [3]. The large majority of B-cell epitopes although they are composed of short linear peptides, are conformational.

Identification of B-cell epitope is considered the main challenging task in the epitope-driven vaccine design [4]. Manual identification of B-cell epitopes by actual experimentations and testing done by scientists is very expensive and has a lot of limitations. Such limitations are (time scale, some experiments can't be done by scientists and ethical concerns). Wherefore, computer based systems can play an 
important role in this task by developing computational methods in predicting B-cell epitopes for scientists.

Computational methods for conformational B-cell epitopes identification require a complete analysis in the context of the native antigen structure however; the linear epitopes only require the sequence of the antigen to be available [5]. Several computational methods have been developed for predicting B-cell epitopes of the both types: linear and conformational epitopes.

There are two major approaches for predicting linear B-cell epitopes; these approaches are propensity scale and mach ine learning methods. The existing methods which rely on propensity scale approach are Parker et al. [5], Karplus et al. [7], Emini et al. [8], PREDITOP [9], PEOPLE [10], BEPITOPE [11] and BcePred [12]; wh ile the recent existing methods which rely on machine learning approach are BepiPred [13], ABCPred [14], Söllner and Mayer [15], Chen et al. [16], Söllner et al. [17], BCPred [18], FBCPred [19], El-Man zala wy et al. [20] and COBEpro [21].

The conformational B-cell epitope prediction methods are also composed of two major approaches; these approaches are sequence and structure based approaches. Sequence based prediction methods try to predict the conformational B-cell epitopes from the antigen primary sequence while in the structure based prediction methods; the antigen 3D structure must be available. The sequence based prediction approach for predicting conformational B-cell epitopes has the advantage that there is no need for the antigen $3 \mathrm{D}$ structure to be available for prediction but only the antigen sequence is enough. CBTOPE [22] is a prediction method relies on the sequence based approach to predict conformational B-cell epitopes from the antigen primary sequence. On the other hand, there are some few methods for predicting conformational B-cell epitopes from the antigen structure; these methods are CEP [23], DiscoTope [3], PEPITO [24], Ellipro [25], EPCES [26], EPSVR [27], EPMeta [27], and Liu $\mathrm{R}$ et al. [28]. Unfortunately, the prediction performances of these methods are still not satisfying and remain far from ideal.

In this paper, we present a different vision for identification of the antigenic epitope sites in the antigen structure chain by predicting the antigen overlapping surface patches that hold the majority of epitope residues in the antigen structure; and thus the scientists can use in vaccine development. From a given antigen structure, the overlapping surface patches are generated, and the surface patch that holds maximum number of epitope residues is considered the epitope patch which is used for vaccination by the scientists. The method is a support vector machine model trained on epitope and non-epitope surface patches generated from antigen structure chains of Pernille et al.'s dataset [3]. The method always choses three top scored paths and treats them as predicted paths. Then the prediction is considered as correct if any of these paths predicts at least $70 \%$ of interacting residues. To evaluate the performance of our model, predictions are made for known structures of an independent test set of antigen chains generated by Po- nomarenko, Ju lia et al. [29]. Additionally, we evaluated our model in terms of the area under receiver operator characteristics curve (AUC) by conducting fivefold cross validation technique on the representative training set collected by Pernille et al. [3]. We co mpared our mode1 with Sharp2 [30]: a server for the prediction of protein-protein interaction sites on the surface of the protein structure. We compared the prediction accuracy of Sharp2 in the benchmark dataset with our model, and it is observed that the identification of the antigenic determinant sites in the protein surfaces using the antigen structural information outperforms the protein-protein interaction method "Sharp2" to predict the antibody interacting sites in the antigen protein surfaces.

\section{Methods}

\subsection{Datas ets}

\subsubsection{Training Dataset}

We obtain $75(\mathrm{Ag}-\mathrm{Ab})$ complexes prepared by Pernille et al. [3] from Discotope supplementary materials. These complexes were selected using X-ray crystallography with resolution less than $3 \AA$. The corresponding antigen PDB file is obtained from Protein Data Bank [31]. Pernille et al. [3] had divided the 75 antigens into 25 heterogeneous groups. The 25 heterogeneous groups of antigens were divided into five data sets for cross validation and testing. In this dataset, a residue in the antigens is determined as epitope residue if the distance between any of its atoms and any atom of antibodies is less than $4 \AA$. This dataset contains 1202 antibody interacting and 13242 non-antibody interacting residues.

\subsubsection{Independent Testset}

We evaluated our model on a Bench mark dataset generated by Ponomarenko et al. [29]. This dataset contains 161 protein chains obtained from 144 (Ag-Ab) complex structures. The antigen residue is considered an epitope residue if the distance between any of its atoms and any atom of antibodies is less than $4 \AA$. We removed sequence redundancy from the 161 antigen chain sequences using CDHIT [32] at $40 \%$ cutoff, obtaining only 50 antigen chains where no two antigen sequences have more than $40 \%$ sequence identity. In order ensure the low sequence identity between training and testing datasets; we removed the chains that already exist in the Ponomarenko et al.'s dataset (our training dataset) and the representative remaining proteins have been selected as testing dataset. Finally we got non redundant test set of 30 antigen chains: 1BGX:T, 1DEE:H, 1E6J:P，1IGC:A，1KEN:A，1NL0:G，1NSN:S，1ORQ:C, 1PKQ:E, 1S78:B, 1SY6:A, 1TZI:V, 1WEJ:F, 1YJD:C, 1YNT:F, 1ZA3:R, 1ZTX:E, 2ADF:A, 1NMB:N, 2B4C:G, 1T03:B, 1R3K:C, 1V7M:V, 1IAI:I, 1IAI:M, 1KB5:A, 1KB5:B, 1QFW:A, 1QFW:B, 1OB1:C. Th is independent test set contains 467 antibody interacting and 6171 non-antibody interacting residues. 


\subsection{Surface Patch Generation Algorithm}

The identification of the protein surface is not an easy task even when the antigen 3-d structure is known. The relative solvent accessibility of a residue in the protein structure is considered a measure of how large the amino acid residue is exposed to the solvent surrounding the protein [33]. NACCESS [34] is a computer program used to compute the atomic accessible surface area of a given 3-dimensional co-ordinate sets (PDB files). For each antigen, the relative accessible surface area is computed and the residues with a relative surface area $\geqslant 5 \%$ are considered the protein surface accessible residues [35]. Each surface accessible residue is used to define a surface patch. A surface patch is composed of the central accessible surface residue followed by $\mathrm{N}$ nearest surface accessible neighbour residues [35], $(\mathrm{N}+1)$ is the patch size. The nearest surface accessible neighbour residues from the patch central accessible surface residue are determined by the Euclidean distance [36] between all surface residues and the patch central residue. Using this procedure, overlapping patches of surface accessible residues are generated from each antigen structure. Figure 1 shows a flow chart of the surface patch generation algorith $\mathrm{m}$.

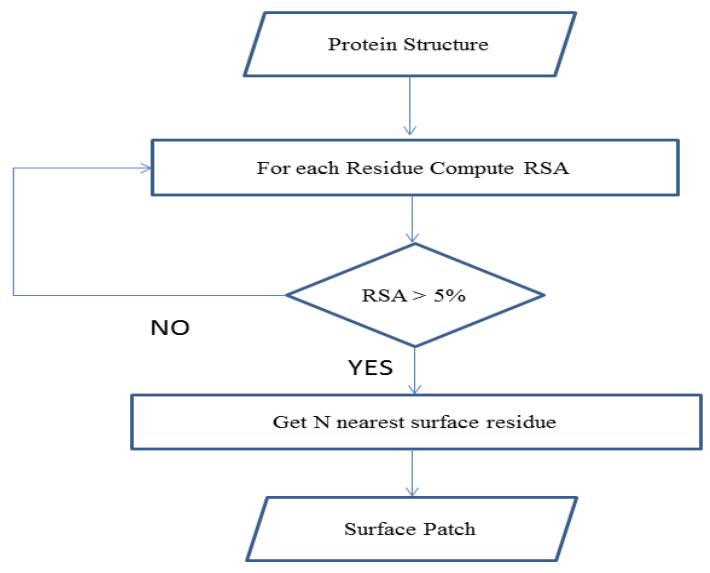

Figure 1. Surface Patch Generation Algorithm

\subsection{Data Prepar ation}

For each antigen structure in the Pernille et al.'s dataset [3], the overlapping surface patches are generated. Ep itope patch is the antigen surface patch that holds the maximum number of epitope surface accessible residues, while non-epitope surface patch is the antigen surface patch that holds the minimum number of epitope surface accessible residues in the antigen structure. Following this rule, the training dataset is composed of 75 and 75 epitope and non-epitope surface patches, respectively. In order to increase the training dataset, we increased the number of non-epitope surface patches for each antigen structure by selecting 6 surface patches holding minimum number of epitope residues, while only one epitope surface patch is generated. For each surface patch, 1 label is assigned to an epitope surface patch and 0 label is assigned to non-epitope surface patch. This training dataset contains a total of 75 epitope surface patches and 450 non-epitope surface patches.

\subsection{Nor malized Relative Solvent Accessibility}

For each surface residue in the antigen structure, the relative solvent accessibility is measured using the program NACCESS [34], and normalized using the following equation:

$$
\mathrm{RSA}_{\text {norm }, \mathrm{r}}=\frac{R S A_{r}-\min (\mathrm{RSA})}{\max (R S A)-\min (R S A)}
$$

where RSA $A_{r}$ is the relative solvent accessibility of residue r; $\max (\mathrm{RSA})$ and $\min (\mathrm{RSA})$ are the maximum and minimum relative solvent accessibility values of all residues in the antigen chain, respectively.

\subsection{Nor malized B-Factor}

Also called "temperature factor", it reflects the flexibility of residues in the protein structure resulting from protein crystallography [37]. For each surface residue in the antigen structure, the B-Factor value is extracted from the antigen 3-dimensional co-ordinate file (PDB file), and normalized using the following equation:

$$
\text { BFactor }_{\text {norm }, \mathrm{r}}=\frac{\text { BFactor }_{\mathrm{r}}-<\text { BFactor }_{\mathrm{r}}>}{\partial\left(\text { BFactor }_{\mathrm{r}}\right)}
$$

Where BFactor $_{\mathrm{r}}$ is the B-Factor of residue $\mathrm{r},<$ BFactor $_{\mathrm{r}}>$ and $\partial\left(\right.$ BFactor $\left._{\mathrm{r}}\right)$ are the mean value and the standard deviation of the B-Factor values of all residues in the antigen chain, respectively.

\subsection{Support Vector Machine Model}

Support vector machine [38] is a classification algorithm aims to find a deterministic mapping function between the input features. Given a set of labeled training patterns (xi, yi), where $x i \in R p$, yi $\in\{+1,-1\}$, training a SVM classifiers involves finding a maximum-margin hyper plane that divides positive and negative training data samples. The hyper plane can be written as $\mathrm{f}(\mathrm{x})=\mathrm{w} . \mathrm{x}+\mathrm{b}$, where "." denotes the dot products, $w$ is a normal vector and $b$ is a bias term. In case of the training data are not linearly separable, a kernel function is used to map the non-linearly separable data into a higher-dimensional space and thus the data are assumed to be linearly separable. Given any two sample observation in the input space (xi, xj), the kernel function can be written as a dot product of two feature vectors into high dimensional feature space $K(x i, x j)=\Phi(x i) T$ $\Phi(x j)$. In this paper, we used Gaussian Radial-Basis Function (RBF) as a kernel function for our support vector machine clas sifier:

$$
K\left(x_{i}, x_{j}\right)=\exp \left(-\frac{\left\|x_{i}-x_{j}\right\|^{2}}{2 \partial^{2}}\right)
$$

where $\partial$ is a parameter.

The support vector machine models had been used in a number of biological applications [39]. We have developed a SVM models using Weka [40] a machine learning workbench. 


\subsection{Epi tope Surface Patches Prediction Alg orithm}

Given the antigen structure, all of its surface residues are generated, and hence all its corresponding surface patches are obtained. The normalized relative solvent accessibility and B-Factor features are calculated for each residue in the surface patch, and hence each surface patch is represented by a vector of dimensions $\mathrm{N} \times 2$ where ( $\mathrm{N}$ is the patch size). A prediction score is associated with each surface patch based on the support vector machine scores for the feature vectors. The top three non-overlapping surface patches with highest prediction scores are generated where no two surface patches have more than $50 \%$ residues overlap Figure 2.Figure 3 shows the flow chart of the prediction algorithm.

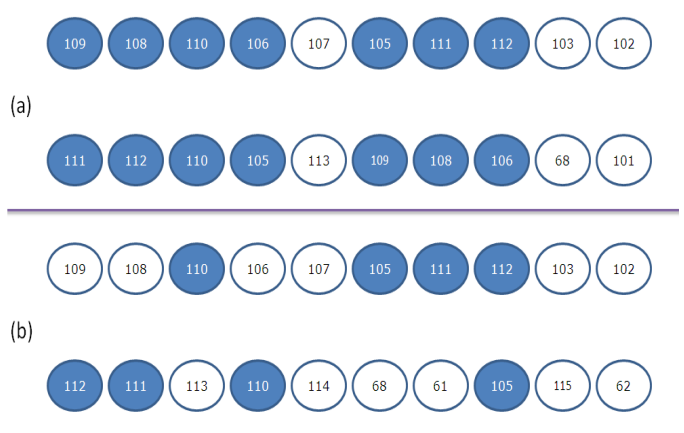

Figure 2. Overlapping/non overlapping surface patches. (a) Overlapping surface patches. (b) Non overlapping surface patches

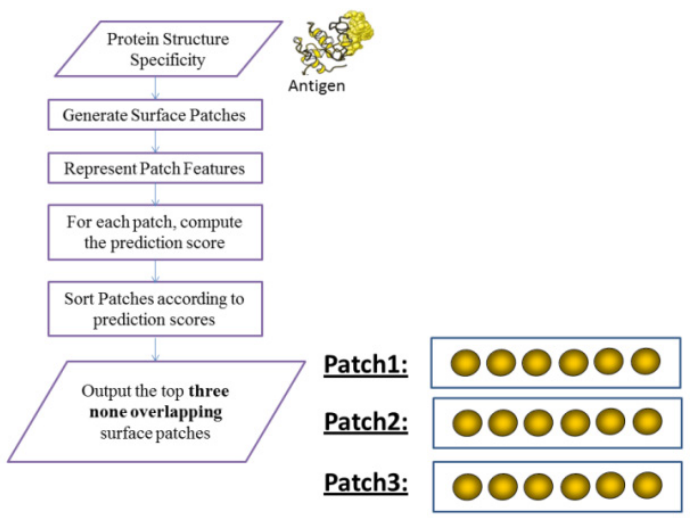

Figure 3. PatchTope Prediction Algorithm

\subsection{Accuracy Me asures Using the Independent Testset}

For each antigen structure chain in the independent test set, all the surface patches are obtained. For each surface patch, the number of epitope residues is calculated. The surface patch which holds the maximum number of ep itope residues over all surface patches generated from the antigen structure is defined as the epitope real surface patch. Figure 4 shows the process of determining the real surface patch.

The top three predicted surface patches are generated using the prediction algorithm. For each predicted surface patch, the relative overlap with the real epitope surface patch is calculated using the following equation:

$$
\text { RelativeOverlap }=\frac{N e_{R} \cap N e_{P}}{N e_{R}} \times 100
$$

where $\mathrm{Ne}_{\mathrm{R}}$ is the number of epitope residues in the real surface patch, and $\mathrm{Ne}_{\mathrm{p}}$ is the number of epitope residues in the predicted surface patch. If the relative overlap for any of the top three predicted surface patches of the antigen structure exceeds $70 \%$, then the prediction is defined to be correct. The prediction accuracy is defined as the ratio of the number of correctly predicted surface patches from the antigen structures to the number of all antigen structures in the independent test set.

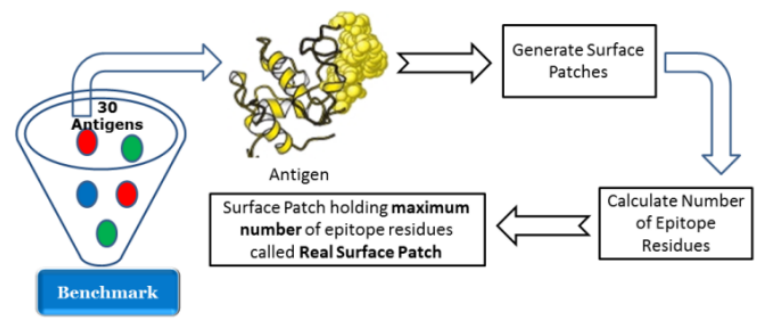

Figure 4. Determining the real surface patch

\section{Results}

\subsection{Analysis of Antibody Inter acting Sites}

In order to understand whether the B-cell epitope residues are located on the surface of the protein structure, we analyzed the Pernille et al.'s dataset to find distributions of the amino acid preference of epitope and non-epitope residues. As shown in Figure 5, most of epitope amino acid residues like Asparagine, Glycine, Arg inine, Lysine, Aspartic, and Threonine are polar (Hydrophilic in nature) while most of the non-epitope amino acid residues like Cysteine, Phenylalanine, Methionine, Alanine, and Tryptophan are hydrophobic (non-polar). It is known that the hydrophobic amino acid residues are not accessible to the solvent while the polar and charged amino acid residues are accessible to the surface of the molecule and are in contact with the solvent [6].

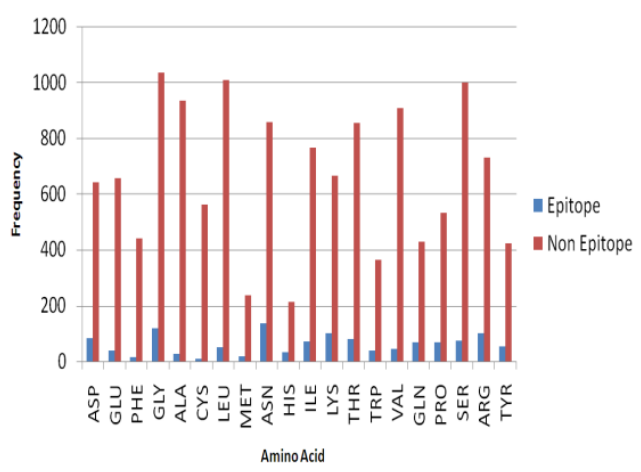

Figure 5. Amino acid preference of epitope and non-epitope residues

The same Pernille et al.'s dataset is analyzed with respect to the antigen surface residues identification, following the role that the antigen residue with relative solvent accessibility $\geqslant 5 \%$ is considered a protein surface accessible residue. The total number of epitope residues in the protein surface and protein body is 1164 and 38 residues, respectively. These findings confirm that most of antigenic epi- 
tope residues are located in the surface of the antigen protein structure.

\subsection{Prediction Results of Fivefold Cross Validation}

Support vector machine model based on Gaussian Radial-Basis Function (RBF) kernel has been developed using the combination of the two antigen structural features (relative solvent accessibility and B-factor). The features are represented by a vector of dimension $\mathrm{Nx} 2$ ( $\mathrm{N}$ is the patch size and equals to 20 residues). The surface patches were generated for each antigen structure in the 75 antigen chains of Pernille et al.'s dataset. Fivefold cross validations were conducted on the five antigen groups of Pernille et al.'s dataset [41]. For each run, one group was left out for testing, while the remaining four groups were used for training. The average area under receiver operator characteris tics curve for the 5 antigen groups reached a maximu m of 0.894 .

\subsection{Prediction of Antigenic Patches in Benchmark Dataset}

The benchmark dataset is used to independently evaluate our model for predicting the antigenic surface patches from the protein antigens. The 75 antigen chains of the Pernille et al.'s dataset are used to train our SVM model, while we predict the antigenic surface patches of the 30 antigen chains generated from the Ponomarenko et al.'s dataset after removing sequence identity. After applying our prediction algorith $m$ it is observed that $70 \%$ of the antigen chains were correctly predicted Table 1 . We obtained an area under receiver operator characteristics curve (AUC) of 0.809 .

Table 1. Results of the prediction algorithm for Benchmark dataset

\begin{tabular}{|c|c|c|c|c|c|c|c|c|c|c|}
\hline \multirow[t]{2}{*}{ PDB Code } & \multirow[t]{2}{*}{ No. Patches ${ }^{\mathrm{a}}$} & \multirow{2}{*}{$\begin{array}{l}\text { Patch } \\
\text { Size }^{b}\end{array}$} & \multicolumn{4}{|c|}{$\begin{array}{c}\text { No. } \\
\text { Epitope Residues }^{\mathrm{c}}\end{array}$} & \multicolumn{3}{|c|}{$\begin{array}{l}\text { \% Relative Overlap } \\
\text { Of top three Patches }\end{array}$} & \multirow[t]{2}{*}{ Pre diction Accu racy } \\
\hline & & & Real & 1 st & 2nd & 3rd & $1 \mathrm{st}$ & 2nd & 3rd & \\
\hline 1BGX:T & 637 & 20 & 15 & 0 & 3 & 8 & 0 & 0 & 0 & Not Correctly Predicted \\
\hline 1DEE:H & 48 & 20 & 11 & 11 & 6 & 7 & 100 & 45 & 55 & Correctly Predicted \\
\hline 1E6J:P & 179 & 20 & 12 & 10 & 9 & 0 & 83 & 75 & 0 & Correctly Predicted \\
\hline 1IAI:I & 185 & 20 & 10 & 10 & 0 & 1 & 100 & 0 & 10 & Correctly Predicted \\
\hline 1IAI:M & 180 & 20 & 6 & 0 & 5 & 0 & 0 & 83 & 0 & Correctly Predicted \\
\hline 1IGC:A & 50 & 20 & 12 & 11 & 3 & 5 & 83 & 25 & 33 & Correctly Predicted \\
\hline 1KB5:A & 90 & 20 & 8 & 7 & 0 & 5 & 88 & 0 & 63 & Correctly Predicted \\
\hline $1 \mathrm{~KB} 5: \mathrm{B}$ & 92 & 20 & 8 & 7 & 8 & 2 & 38 & 100 & 25 & Correctly Predicted \\
\hline 1KEN:A & 245 & 20 & 10 & 0 & 10 & 0 & 0 & 100 & 0 & Correctly Predicted \\
\hline $1 \mathrm{NL} 0: \mathrm{G}$ & 32 & 20 & 5 & 5 & 0 & 2 & 100 & 0 & 40 & Correctly Predicted \\
\hline 1NMB:N & 243 & 20 & 15 & 0 & 0 & 0 & 0 & 0 & 0 & Not Correctly Predicted \\
\hline 1NSN:S & 112 & 20 & 10 & 8 & 9 & 5 & 50 & 50 & 10 & Not Correctly Predicted \\
\hline 1OB1:C & 89 & 20 & 10 & 8 & 7 & 3 & 80 & 40 & 0 & Correctly Predicted \\
\hline 1ORQ:C & 205 & 20 & 10 & 0 & 0 & 10 & 0 & 0 & 100 & Correctly Predicted \\
\hline 1PKQ:E & 95 & 20 & 14 & 8 & 12 & 3 & 50 & 86 & 7 & Correctly Predicted \\
\hline 1QFW:A & 87 & 20 & 8 & 8 & 3 & 0 & 100 & 0 & 0 & Correctly Predicted \\
\hline 1QFW:B & 108 & 20 & 12 & 0 & 0 & 12 & 0 & 0 & 100 & Correctly Predicted \\
\hline 1R3K:C & 88 & 20 & 13 & 5 & 7 & 6 & 38 & 46 & 46 & Not Correctly Predicted \\
\hline 1S78:B & 431 & 20 & 9 & 0 & 0 & 0 & 0 & 0 & 0 & Not Correctly Predicted \\
\hline 1SY6:A & 140 & 20 & 10 & 8 & 0 & 0 & 80 & 0 & 0 & Correctly Predicted \\
\hline 1T03:B & 341 & 20 & 9 & 0 & 0 & 0 & 0 & 0 & 0 & Not Correctly Predicted \\
\hline 1TZI:V & 90 & 20 & 3 & 0 & 0 & 3 & 0 & 0 & 100 & Correctly Predicted \\
\hline 1V7M:V & 113 & 20 & 14 & 11 & 12 & 6 & 79 & 64 & 36 & Correctly Predicted \\
\hline 1WEJ:F & 93 & 20 & 10 & 8 & 8 & 0 & 80 & 80 & 0 & Correctly Predicted \\
\hline 1YJD:C & 97 & 20 & 9 & 0 & 0 & 8 & 0 & 0 & 44 & Not Correctly Predicted \\
\hline 1YNT:F & 192 & 20 & 13 & 0 & 4 & 12 & 0 & 15 & 62 & Not Correctly Predicted \\
\hline $1 \mathrm{ZA} 3: \mathrm{R}$ & 85 & 20 & 11 & 0 & 9 & 8 & 0 & 73 & 64 & Correctly Predicted \\
\hline 1ZTX:E & 81 & 20 & 11 & 11 & 10 & 0 & 91 & 73 & 0 & Correctly Predicted \\
\hline 2ADF:A & 430 & 20 & 8 & 0 & 2 & 0 & 0 & 13 & 0 & Not Correctly Predicted \\
\hline 2B4C:G & 275 & 20 & 11 & 10 & 4 & 6 & 82 & 36 & 45 & Correctly Predicted \\
\hline
\end{tabular}

${ }_{\mathrm{b}}^{\mathrm{a}}$ Total number of surface pat ches of each antigen chain.

${ }^{b}$ Number of residues in the surface patch.

c Number of epitope residues in the real epitope patch and the top three non-overlapping surface patches generated from our prediction algorithm.

d Relative overlap between the top three surface pat ches and the real surface patch.

${ }^{\mathrm{e}}$ The prediction accuracy, if the relative overlap for any of the top three predicted surface patches of the antigen structure exceeds $70 \%$, then the prediction is defined to be correct. 


\subsection{Comparisons with a Protein-Protein Interaction Server}

Sharp2 [30] is a web server for pred icting protein-protein interaction sites on the surface of the 3D structure of a protein. The protein interacting sites may be an identical protein, a different protein that is larger, a different protein that is smaller, or an antibody. The user friendly web server enables the scientists to choose the protein interacting sites, and hence the algorithm parameters for predicting protein-protein interaction sites are changed accordingly[35]. For each antigen chain in the benchmark dataset, the protein interacting surface patches have been downloaded using Sharp2 web server with parameters: (protein type = Type D; Interacting partner is an antibody; and patch size $=20$ ). The surface patches predicted from the antigen chain are ranked based on the patches with highest combined scores. The top three non-overlapping surface patches with highest combined scores are generated where no two surface patches have more than $50 \%$ residues overlap. For each antigen chain in the benchmark dataset, the relative overlap of each surface patch in the top three non-overlapping surface patches with the real epitope surface patch is calculated. We observed that only $28 \%$ of the antigen chains were correctly predicted (Table 2). Comparing this prediction accuracy in the Ponomarenko et al.'s dataset with our model reveals that identifying the antigenic determinant sites in the protein surfaces using the antigen structural information outperforms the traditional protein-protein interaction algorithms to predict the interacting sites in the protein surfaces.

Table 2. Results of the prediction of protein-protein interaction sites using Sharp2 on the Benchmark dataset

\begin{tabular}{|c|c|c|c|c|c|c|c|c|c|c|}
\hline \multirow[t]{2}{*}{ PDB Code } & \multirow{2}{*}{$\begin{array}{c}\text { No. } \\
\text { Patches }^{\mathrm{a}}\end{array}$} & \multirow{2}{*}{$\begin{array}{l}\text { Patch } \\
\text { Size }^{b}\end{array}$} & \multicolumn{4}{|c|}{$\begin{array}{c}\text { No. } \\
\text { Epitope Resi dues }^{\mathrm{c}}\end{array}$} & \multicolumn{3}{|c|}{$\begin{array}{l}\% \text { Relative O verlap } \\
\text { Of top three Patches }\end{array}$} & \multirow[t]{2}{*}{ Prediction Accuracy $^{\mathrm{e}}$} \\
\hline & & & Real & 1st & 2nd & 3rd & 1 st & 2nd & 3rd & \\
\hline 1BGX:T & 637 & 20 & 15 & 0 & 7 & 4 & 0 & 0 & 0 & Not Correctly Predicted \\
\hline 1DEE:H & 48 & 20 & 11 & 2 & 5 & 7 & 18 & 45 & 55 & Not Correctly Predicted \\
\hline 1E6J:P & 179 & 20 & 12 & 0 & 0 & 2 & 0 & 0 & 17 & Not Correctly Predicted \\
\hline 1IAI:I & 185 & 20 & 10 & 0 & 0 & 0 & 0 & 0 & 0 & Not Correctly Predicted \\
\hline 1IAI:M & 180 & 20 & 6 & 0 & 0 & 0 & 0 & 0 & 0 & Not Correctly Predicted \\
\hline 1IGC:A & 50 & 20 & 12 & 10 & 3 & 11 & 83 & 17 & 92 & Correctly Predicted \\
\hline 1KB5:A & 90 & 20 & 8 & 5 & 1 & 8 & 50 & 13 & 100 & Correctly Predicted \\
\hline $1 \mathrm{~KB} 5: \mathrm{B}$ & 92 & 20 & 8 & 1 & 2 & 7 & 0 & 25 & 25 & Not Correctly Predicted \\
\hline $1 \mathrm{KEN}: \mathrm{A}$ & 245 & 20 & 10 & 2 & 0 & 0 & 0 & 0 & 0 & Not Correctly Predicted \\
\hline 1NL0:G & 32 & 20 & 5 & 5 & 2 & 5 & 100 & 40 & 100 & Correctly Predicted \\
\hline 1NMB:N & 243 & 20 & 15 & 0 & 0 & 0 & 0 & 0 & 0 & Not Correctly Predicted \\
\hline $1 \mathrm{NSN}: \mathrm{S}$ & 112 & 20 & 10 & 5 & 3 & 6 & 20 & 20 & 50 & Not Correctly Predicted \\
\hline $1 \mathrm{OB} 1: \mathrm{C}$ & 89 & 20 & 10 & 0 & 0 & 8 & 0 & 0 & 40 & Not Correctly Predicted \\
\hline 1ORQ:C & 205 & 20 & 10 & 0 & 0 & 0 & 0 & 0 & 0 & Not Correctly Predicted \\
\hline 1PKQ:E & 95 & 20 & 14 & 7 & 2 & 14 & 50 & 14 & 100 & Correctly Predicted \\
\hline 1QFW:A & 87 & 20 & 8 & 3 & 3 & 1 & 0 & 0 & 0 & Not Correctly Predicted \\
\hline $1 \mathrm{QFW}: \mathrm{B}$ & 108 & 20 & 12 & 0 & 0 & 12 & 0 & 0 & 100 & Correctly Predicted \\
\hline 1R3K:C & 88 & 20 & 13 & 11 & 0 & 0 & 77 & 0 & 0 & Correctly Predicted \\
\hline 1S78:B & 431 & 20 & 9 & 0 & 0 & 9 & 0 & 0 & 56 & Not Correctly Predicted \\
\hline 1SY6:A & 140 & 20 & 10 & 2 & 0 & 10 & 10 & 0 & 100 & Correctly Predicted \\
\hline 1T03:B & 341 & 20 & 9 & 6 & 0 & 0 & 56 & 0 & 0 & Not Correctly Predicted \\
\hline 1TZI:V & 90 & 20 & 3 & 0 & 0 & 2 & 0 & 0 & 33 & Not Correctly Predicted \\
\hline $1 \mathrm{~V} 7 \mathrm{M}: \mathrm{V}$ & 113 & 20 & 14 & 2 & 0 & 2 & 14 & 0 & 14 & Not Correctly Predicted \\
\hline 1WEJ:F & 93 & 20 & 10 & 1 & 1 & 0 & 10 & 10 & 0 & Not Correctly Predicted \\
\hline 1YJD:C & 97 & 20 & 9 & 2 & 5 & 0 & 0 & 56 & 0 & Not Correctly Predicted \\
\hline 1YNT:F & 192 & 20 & 13 & 0 & 0 & 0 & 0 & 0 & 0 & Not Correctly Predicted \\
\hline $1 \mathrm{ZA} 3: \mathrm{R}$ & 85 & 20 & 11 & 0 & 0 & 0 & 0 & 0 & 0 & Not Correctly Predicted \\
\hline 1ZTX:E & 81 & 20 & 11 & 10 & 11 & 7 & 82 & 82 & 55 & Correct ly Predicted \\
\hline 2ADF:A & 430 & 20 & 8 & 0 & 0 & 0 & 0 & 0 & 0 & Not Correctly Predicted \\
\hline 1BGX:T & 637 & 20 & 15 & 0 & 7 & 4 & 0 & 0 & 0 & Not Correctly Predicted \\
\hline
\end{tabular}

${ }_{\mathrm{b}}^{\mathrm{a}}$ Total number of surface pat ches of each antigen chain.

bumber of residues in the surface patch.

${ }^{c}$ Number of epitope residues in the real epitope patch and the top three non-overlapping surface patches generated from our prediction algorithm.

${ }^{d}$ Relative overlap between the top three surface pat ches and the real surface pat ch.

e The prediction accuracy, if the relative overlap for any of the top three predicted surface patches of the antigen structure exceeds $70 \%$, then the prediction is defined to be correct. 


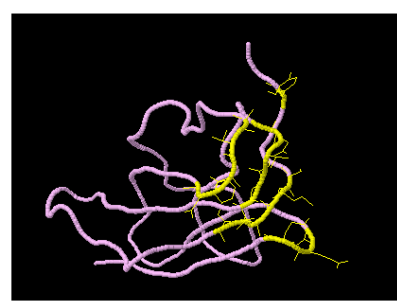

(a)

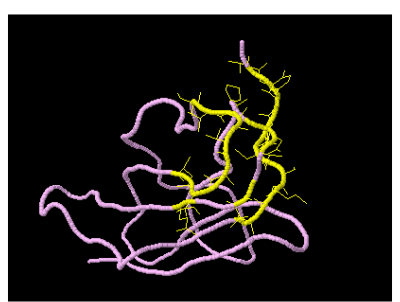

(b)
Figure 6. Visualization of prediction results from complex (PDB ID: 1ZTX, Chain ID: E)(a) Actual epitope residues determined in the benchmark epitope dataset. (b) Predicted epitope surface patch

\subsection{Visualization of Predicted Patches for an Example}

To illustrate the effectiveness of our method, we choose a chain complex (PDB ID: 1ZTX, Chain ID: E) from the benchmark dataset as an example to visualize the predicted surface patch which holds the maximum number of ep itope residues in the chain complex. We compare the residues of the predicted epitope surface patch with the actual epitope residues determined in the benchmark epitope dataset. (Figure 6) shows that the predicted surface patch identified by our classifier holds most of the epitope residues in the protein complex structure (11 out of 16 epitope residues). The predicted surface patch can then be used in vaccine development.

\subsection{Patch Tope Implementation}

PatchTope is a user friendly web-based bioinformatics tool for the prediction of the antigenic epitope surface patches which hold the most epitope residues in the given antigen protein structure. The server is developed using Java Servlet and HTML. The user may submit the antigen structure by entering its PDB-Id or uploading a structure file in a PDB format. Moreover, the user may enter the chain Id for the protein chain of interest and then click on submit button. For the given antigen structure, the surface accessible residues are extracted, and each one defines a surface patch. For each surface patch, the feature vector is generated by computing the relative solvent accessibility of each residue using NACCESS [34] program, and extracting the B-Factor feature from the PDB file. The generated surface patches are then passed as input to the trained support vector machine model (one by one). The top three non-overlapping surface patches with highest prediction scores are generated where no two surface patches have more than $50 \%$ residues overlap. $3 \mathrm{D}$ view of the antigen structure is generated using JMOL [42] and the predicted surface patch residues are marked as yellow color for each predicted surface patch according to the user selection. PatchTope requires Netscape v6.0 or Internet Exp lorer v 6.0 or higher and Java script enabled. The web-server is freely available at http://www.fci.cu.edu.eg:8080/PatchTope/.

\section{Conclusions}

In this paper we propose a new computational method for predicting antigenic surface patches that interact with B-cell. Computing the relative overlapping of predicted patches with the real epitope patch in known structures of independent test set showed that structural information of the antigen chains can be used in predicting the protein interacting sites on the surface of the protein structure. Compared with popular prediction methods for predicting protein-protein interaction using patch analysis, our approach showed better performance in terms of prediction accuracy.

\section{ACKNOWLEDGEMENTS}

It is a pleasure to thank Dr: Yasser EL-Manzalawy for his great support and help. He provided encouragement, sound advice, and lots of good ideas during the long useful discussions which made this work possible.

Additionally, we thank the faculty of computers and information, Cairo University for publishing PatchTope web server in its domain.

\section{REFERENCES}

[1] Kolaskar AS, Tongaonkar PC. A semi-empirical method for prediction of antigenic determinants on protein antigens. FEBS Lett. 1990 Dec 10;276(1-2):172-4

[2] Ben Berkhout. Evolution of Live-Attenuated HIV Vaccines. BioPharm International Supplements, Volume 24, pp. s4-s8, Jan 2, 2011.

[3] Ole Lund, Morten Nielsen, Claus Lundegaard, Can Kesmir and SørenBrunak. Immunological Bioinformatics. ISBN-10: 0-262-12280-4 ISBN-13: 978-0-262-12280-1, September 2005.

[4] Andersen PH, Nielsen M, Lund O: Prediction of residues in discontinuous B-cell epitopes using protein 3D structures. Protein Science 2006, 15:2558-2567.

[5] EL-Manzalawy, Yasser and Honavar, Vasant. Recent advances in B-cell epitope prediction methods. Immunome Research 201 0, 6(Supp12):S2doi:10.1186/1745-7580-6-S2-S 2.

[6] Parker JM, Guo D, Hodges RS. New hydrophilicity scale derived from high-performance liquid chromatography peptide retention data: correlation of predicted surface residues with antigenicity and X-ray-derived accessible sites. Biochemistry. 1986 Sep 23;25

[7] Laurence Lins, Annick Thomas, and Robert Brasseur. Anal$\mathrm{y}$ sis of accessible surface of residues in proteins. Protein Sci, Vol. 12, No. 7. (July 2003), pp. 1406-1417.

[8] Karplus PA, Schulz GE. Prediction of Chain Flexibility in Proteins - A tool for the Selection of Peptide Antigens. Naturwissenschafren $1985 ; 72: 212-3$

[9] Emini EA, Hughes JV, Perlow DS, Boger J. Induction of hepatitis A virus-neutralizing antibody by a virus-specific 
synthetic peptide. J Virol. 1985 Sep;55(3):836-9.

[10] J. Pellequer and E. Westhof. PREDITOP: a program for antigenicity prediction. J Mol Graph, 11:204-210, 1993.

[11] A. Alix. Predictive estimation of protein linear epitopes by using the program PEOPLE. Vaccine, 18:311-4, 1999.

[12] M. Odorico and J. Pellequer. BEPITOPE: predicting the location of continuous epitopes and patterns in proteins. $\mathrm{J}$ MolRecognit, 16:20-22, 2003.

[13] S. Saha and G. Raghava. BcePred: Prediction of continuous B-cell epitopes in antigenic sequences using physicochemical properties. Artificial Immune Systems, Third International Conference (ICARIS 2004), LNCS, 3239:197-204, 2004.

[14] Jens Erik Pontoppidan Larsen, Ole Lund and Morten Nielsen. Improved method for predicting linear B-cell epitopes. Immunome Research 2006, 2:2doi:10.1186/1745-7580-2-2.

[15] Saha S, Raghava G. Prediction of continuous B-cell epitopes in an antigen using recurrent neural network. Proteins. 2006;65:40-48. doi: 10.1002/prot. 21078 .

[16] Söllner J, Mayer B. Machine learning approaches for prediction of linear B-cell epitopes onproteins. J. 2006;19:200-208. doi: 10.1002/jmr.771.

[17] Chen J, Liu H, Yang J, Chou K. Prediction of linear B-cell epitopes using amino acid pair antigenicity scale. Amino Acids. 2007;33:423-428. doi: 10.1007/s00726-006-0485-9

[18] Söllner J, Grohmann R, Rapberger R, Perco P, Lukas A, Mayer B, Blythe M. Analysis and prediction of protective continuous B-cell epitopes on pathogen proteins. Immunome Res. 2008;7:4.

[19] EL-Manzalawy Y, Dobbs D, Honavar V. Predicting linear B-cell epitopes using string kernels. J. 2008;21:243-255. doi: 10.1002/jmr.893.

[20] EL-Manzalawy Y, Dobbs D, Honavar V. Predicting flexible length linear B-cell epitopes. 7th International Conference on Computational Systems Bioinformatics. 2008. pp. 121-131.

[21] EL-Manzalawy Y, Dobbs D, Honavar V. Predicting linear B-cell epitopes using evolutionary information. IEEE International, Conference on Bioinformatics and Biomedicine. 2008.

[22] Sweredoski M, Baldi P. COBEpro: a novel system for predicting continuous B-cell epitopes. Protein Eng Des Sel. 2009;22(3):113-120. doi: 10.1093/protein/gzn075.

[23] HifzurRahman Ansari and Gajendra PS Raghava: Identification of conformational B-cell Epitopes in an antigen from its primary sequence. Immunome Research 2010, 6:6doi:10.1186/1745-7580-6-6.

[24] Kulkarni-Kale U, Bhosle S, Kolaskar A. CEP: a conformational epitope prediction server. Nucleic Acids Res. 2005;33:W168. doi: 10.1093/nar/gki460.

[25] Sweredoski M, Baldi P. PEPITO: improved discontinuous B-cell epitope prediction using multiple distance thresholds and half sphere exposure. Bioinformatics. 2008;24(12):1459-1460. doi: 10.1093/bioinformatics/btn199.
[26] Ponomarenko J, Bui H, Li W, Fusseder N, Bourne P, Sette A, Peters B. ElliPro: a new structure-based tool for the prediction of antibody epitopes. BMC bioinformatics. 2008;9:514. doi: 10.1186/1471-2105-9-514.

[27] Liang S, Zheng D, Zhang C, Zacharias M. Prediction of antigenic epitopes on protein surfaces by consensus scoring. BMC Bioinformatics. 2009;10: 302

[28] Liang S, Zheng D, Standley D, Yao B, Zacharias M, and Zhang, C. EPSVR and EPMeta: prediction of antigenic epitopes using support vector regression and multiple server results. BMC Bioinformatics 2010, 11:381. doi: 10.1186/1471-2105-11-381.

[29] Liu R, Hu J (2011) Prediction of Discontinuous B-Cell Epitopes Using Logistic Regression and Structural Information. J Proteomics Bioinform 4: 010-015. doi:10.4172/jpb.1000161.

[30] Ponomarenko JV, Bourne PE: Antibody-protein interactions: benchmark datasets and prediction tools evaluation. BMC StructBiol 2007, 7:64.

[31] Murakami,Y. \& Jones, S. (2005) SHARP2 ; Protein-Protein Predictions using Patch Analysis. Bioinformatics, submitted.

[32] Berman HM, Westbrook J, Feng Z, Gilliland G, Bhat TN, et al. (2000) The protein data bank. Nucleic Acids Res 28: 235-242.

[33] Li W, Godzik A: Cd-hit: a fast program for clustering and comparing large sets of protein or nucleotide sequences. Bioinformatics 2006, 22:1658-1659.

[34] H. Chen, H. Zhou, X. Hu, and I. Yoo, Classification Comparison of Prediction of Solvent Accessibility From Protein Sequences, in Proc. APBC, 2004, pp.333-338.

[35] Hubbard, S.J. and Thornton, J.M. 1993. NACCESS computer program. Department of Biochemistry and Molecular Biology, University College of London, UK.

[36] Jones, S and Thornton, JM (1997) Analy sis of Protein-Protein Interaction Sites using Surface Patches. Journal of Molecular Biology , 272 (1) 121 - 132.

[37] http://en.wikipedia.org/wiki/Euclidean_distance

[38] T E Creighton. Proteins: Structures and molecular properties. pp 515. W H Freeman, New York. 1983. £33.75 ISBN 0-7167-1566-X.

[39] Vapnik V. 2000. The Nature of Statistical Learning Theory, 2nd Ed. Springer-Verlag New York, Inc. New York, NY, USA.

[40] ZhengRong Yang. Biological applications of support vector machines, Brief Bioinform (2004) 5(4): 328-338 doi:10.1093/bib/5.4.328.

[41] Mark Hall, Eibe Frank, Geoffrey Holmes, Bernhard Pfahringer, Peter Reutemann, Ian H. Witten (2009); The WEKA Data Mining Software: An Update; SIGKDD Explorations, Volume 11, Issue 1.

[42] http://www.cbs.dtu.dk/suppl/immunology/DiscoTope.php

[43] http://jmol.sourceforge.net/ 